M. Yasumoto

Nagoya Math. J.

Vol. 105 (1987), 33-37

\title{
NONSTANDARD ARITHMETIC OF POLYNOMIAL RINGS
}

\author{
MASAHIRO YASUMOTO \\ Dedicated to Professor Toshiyuki Tugué on his 60th birthday
}

Let $f\left(X, T_{1}, \cdots, T_{m}\right)$ be a polynomial over an algebraic number field $K$ of finite degree. In his paper [2], T. Kojima proved

Theorem. Let $K=\boldsymbol{Q}$. If for every $m$ integers $t_{1}, \cdots, t_{m}$, there exists an $r \in K$ such that $f\left(r, t_{1}, \cdots, t_{m}\right)=0$, then there exists a rational function $g\left(T_{1}, \cdots, T_{m}\right)$ over $\boldsymbol{Q}$ such that

$$
f\left(g\left(T_{1}, \cdots, T_{m}\right), T_{1}, \cdots, T\right)=0 .
$$

Later, A. Schinzel [6] proved

THEOREM. If for every $m$ arithmetic progressions $P_{1}, \cdots, P_{m}$ in $Z$ there exist integers $t_{i} \in P_{i}(i \leqq m)$ and an $r \in K$ such that $f\left(r, t_{1}, \cdots, t_{m}\right)=0$ then there exists a rational function $g\left(T_{1}, \cdots, T_{m}\right)$ over $K$ such that

$$
f\left(g\left(T_{1}, \cdots, T_{m}\right), T_{1}, \cdots, T_{m}\right)=0 .
$$

In his thesis [7], S. Tung applied these theorems to solve some dicidability and definability problems. In this paper, we are concerned with geometric progressions of values of $T_{1}, \cdots, T_{m}$. We prove

Theorem 1. Assume that there exists $a_{1}, \cdots, a_{m} \in K$ other than 0 and roots of unity such that for any $m$ integers $t_{1}, \cdots, t_{m}$, there exists an $r \in K$ with $f\left(r, a_{1}^{t_{1}}, \cdots, a_{m}^{t_{m}}\right)=0$. Then there exist a rational function $g\left(T_{1}, \cdots, T_{m}\right)$ over $K$ and $m$ integers $k_{1}, \cdots, k_{m}$ not more than $k$ such that

$$
f\left(g\left(T_{1}, \cdots, T_{m}\right), T_{1}^{k_{1}}, \cdots, T_{m}^{k_{m}}\right)=0
$$

where $k$ is the $X$-degree of $f\left(X, T_{1}, \cdots, T_{m}\right)$.

$\S 1$.

In case of $m=1$, Theorem 1 is an easy consequence from Theorem of P. Roquette (Theorem 2.1 [4]) as follows.

Received July 8, 1985. 
Let $\omega \epsilon^{*} N-N$ be a nonstandard natural number which is divisible by all natural number where ${ }^{*} N$ is an enlargement of $N$. By the assumption of Theorem 1 , there exists a $\delta \in{ }^{*} K$ such that

$$
f\left(\delta, a^{\omega}\right)=0 .
$$

Let $k_{1}=\left[K\left(\delta, a^{\omega}\right) ; K\left(a^{\omega}\right)\right]$. Since the $X$-degree of $f(X, T)$ is $k$,

$$
k_{1} \leqq k \text {. }
$$

According to Theorem 2.1 in [4], we have

Theorem 2. For each natural number $n$, there is one and only one extension $F_{n}=K\left(a^{\omega / n}\right)$ of $K\left(a^{\omega}\right)$ within ${ }^{*} K$ such that

$$
\left[F_{n} ; K\left(a^{\omega}\right)\right]=n
$$

where ${ }^{*} K$ is an enlargement of $K$.

Hence, $K\left(\delta, a^{\omega}\right)=K\left(a^{\omega / k_{1}}\right)$. Therefore there exists a rational function $g(T)$ over $T$ such that $\delta=g\left(a^{\omega / k_{1}}\right)$. Now we have

$$
f\left(g\left(a^{\omega / k_{1}}\right), a^{\omega}\right)=0 \text {. }
$$

Since $a^{\omega / k_{1}}$ is transcendental over $K$,

$$
f\left(g(T), T^{k_{1}}\right)=0
$$

as contended.

\section{$\S 2$.}

In this section we prove Theorem 1 for the case $m=2$. To prove it, we need iterated enlargements. Iterated enlargements are very useful method but sometime they may cause confusion. So first we discuss basic properties of iterated enlargements. Let ${ }^{\circ} K$ be an enlargement of $K$. We consider the structure $\left({ }^{\circ} K, K\right)$ and its enlargement $*\left({ }^{\circ} K, K\right)=\left({ }^{\circ} K,{ }^{*} K\right)$. Then ${ }^{* \circ} K$ is an elementary extension of $* K$ but not an enlargement of ${ }^{*} K$. By Theorem of Roquette, for each $n \in N$ and $a \in K$ other than 0 and roots of unity, the following statement is valid for $\left({ }^{\circ} K, K\right)$;

"For each $\omega \in{ }^{\circ} N-N$, there is one and only one extension $F_{n}$ of $K\left(a^{\omega}\right)$ within ${ }^{\circ} K$ such that $\left[F_{n} ; K\left(a^{\omega}\right)\right]=n . "$

By nonstandard principle, the above statement holds for $\left({ }^{\circ} K,{ }^{*} K\right)$; 
"For each $\omega \in{ }^{* \circ} N-{ }^{*} N$, there is one and only one extension $F_{n}$ of $L$ within ${ }^{* \circ} K$ such that $\left[F_{n} ; L\right]=n$."

where $L=\left\{h\left(a^{\omega}\right) \mid h(X) \in *(K(X))\right\}$. It should be noted that the rational function field over $* K$ in the sence of the enlargement generated by $a^{\omega}$ must be $L$, not $* K\left(a^{\omega}\right)$.

Remark. ${ }^{* \circ} K$ is an enlargement of ${ }^{\circ} K$, but Theorem 2 (replacing ${ }^{\circ} K$ and $K$ by ${ }^{* \circ} K$ and ${ }^{\circ} K$ respectively) does not hold, because ${ }^{* \circ} N$ is not an end extension of ${ }^{\circ} N$, namely there exist a $c \in{ }^{*} N-{ }^{\circ} N$ and a $d \in{ }^{\circ} N$ with $c<d$. In fact, let $c \in^{* \circ} N$ be an element which satisfies the set of formulas $T=\left\{c<d \mid d \in{ }^{\circ} N-N\right\} \cup\{n<c \mid n \in N\}$. Since any finite subset of $T$ is satisfiable and ${ }^{*} N$ is an enlargement of ${ }^{\circ} N$, such $c$ exists. On the other hand, ${ }^{\circ} N$ is an end extension of $N$, so ${ }^{*} N$ is also an end extension of $* N$, therefore ${ }^{*} K$ is not an enlargement of $* K$.

The following Lemma 1 has been proved in [4] but we include its proof for the convenience of the reader.

LEMMA 1. Let $M$ be any field. Then $* M(X)$ is relatively algebraically closed in $*(M(X))$.

Proof. Let $u(X) / v(X)$ be any element of $*(M(X))-* M(X)$ where $u(X)$, $v(X) \in^{*}(M[X])$ and g.c.d. $(u(X), v(X))=1$ and assume that $u(X) / v(X)$ is algebraic over $* M(X)$. Then there exist $c_{0}, c_{1}, \cdots, c_{n} \in *^{*} M[X]$ with $c_{0} \neq 0$ and $c_{0}(u / v)^{n}+c_{1}(u / v)^{n-1}+\cdots+c_{n}=0$. Since $u / v \notin * M(X)$, the degree of $u$ or $v$ is infinitely large. We may assume without loss of generality that the degree of $v$ is infinitely large. Then

$$
\begin{aligned}
& c_{0} u^{n}+c_{1} u^{n-1} v+\cdots+c_{n} v^{n}=0 \\
& c_{0} u^{n} \equiv 0 \quad \bmod (v) .
\end{aligned}
$$

Since g.c.d. $(u, v)=1$,

$$
c_{0} \equiv 0 \quad \bmod (v)
$$

Since the degree of $v$ is infinitely large and the degree of $c_{0}$ is finite, $c_{0}$ $=0$. This is a contradiction.

Lemma 2. Let $a \in K$ be not 0 nor roots of unity and $\omega \in{ }^{* \circ} N-{ }^{*} N$ be divisible by all natural number. Then $* K\left(a^{\omega / n}\right)$ is the unique extension of $* K\left(a^{\omega}\right)$ of degree $n$ within ${ }^{* \circ} K$.

Proof. Let $x \in{ }^{* \circ} K$ be algebraic over ${ }^{*} K\left(a^{\omega}\right)$ of degree $n$. Then $x \in$ $L\left(a^{\omega / n}\right)$ because $L\left(a^{\omega / n}\right)$ is the unique extension of $L$ of degree $n$ within ${ }^{*}{ }^{\circ} K$ 
and ${ }^{*} K\left(a^{\omega}\right)$ is relatively algebraically closed in $L=\left\{h\left(a^{\omega}\right) \mid h(X) \epsilon^{*}(K(X))\right\}$ by Lemma 1.

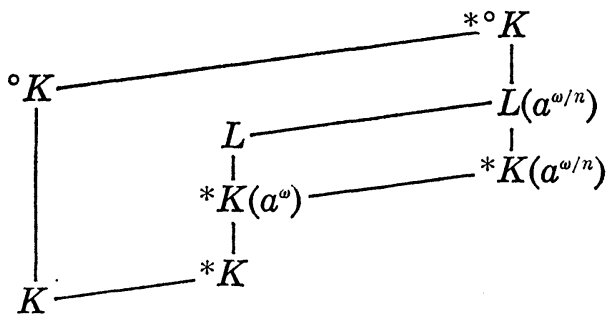

Again by Lemma $1,{ }^{*} K\left(a^{\omega / n}\right)$ is relatively algebraically closed in $L\left(a^{\omega / n}\right)=$ $\left\{h\left(a^{\omega / n}\right) \mid h(X) \in *(K(X))\right\}$. Hence $x \in * K\left(a^{\omega / n}\right)$, as contended.

Let $\omega \in{ }^{* \circ} N-{ }^{*} N$ and $\mu \in *^{*} N-N$ be divisible by all natural numbers. By the assumption of Theorem 1 , there exists a $\delta \in{ }^{*} K$ with

$$
f\left(\delta, a_{1}^{\omega}, a_{2}^{\mu}\right)=0 .
$$

Since $a_{2}^{\mu} \in{ }^{*} K, \delta$ is algebraic over $* K\left(a_{1}^{\omega}\right)$ of degree $k_{1} \leqq k$. Hence by Lemma $2, \delta \in{ }^{*} K\left(a_{1}^{\omega / k_{1}}\right)$. Let $F$ be the relative algebraic closure of $K\left(a_{2}^{\mu}\right)$ within ${ }^{*} K$. Then $\delta \in F\left(a_{1}^{\omega / k_{1}}\right)$ because $F\left(a_{1}^{\omega / k_{1}}\right)$ is relatively algebraically closed in $* K\left(a_{1}^{\omega / k_{1}}\right)$. By Theorem 2, $K\left(a_{2}^{\mu}\right)$ has the unique extension $K\left(a_{2}^{\mu / n}\right)$ of degree $n$ within $F$. Since $a_{1}^{\omega / k_{1}}$ is transcendental over $F, K\left(a_{1}^{\omega / k_{1}}, a_{2}^{\mu}\right)$ has the unique extension $K\left(a_{1}^{\omega / k_{1}}, a_{2}^{\mu / n}\right)$ of degree $n$ within $F\left(a_{1}^{\omega / k_{1}}\right)$.

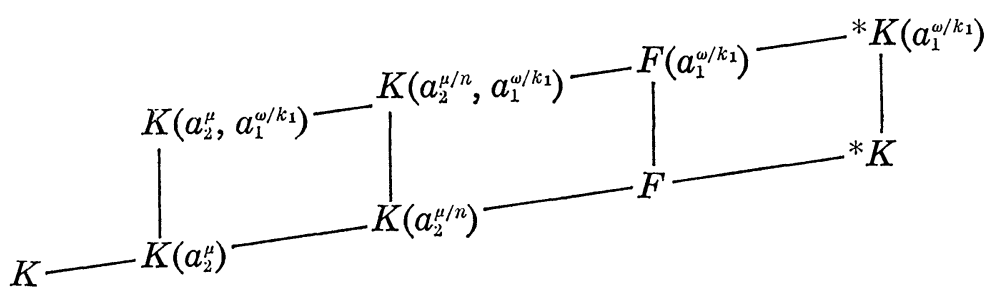

Let $k_{2}=\left[K\left(\delta, a_{2}^{\mu}, a_{1}^{\omega / k_{1}}\right) ; K\left(a_{2}^{\mu}, a_{1}^{\omega / k_{1}}\right)\right]$. Then $k_{2} \leqq k$ and

$$
K\left(\delta, a_{2}^{\mu}, a_{1}^{\omega / k_{1}}\right)=K\left(a_{2}^{\mu / k_{2}}, a_{1}^{\omega / k_{1}}\right) .
$$

Hence there exists a rational function $g\left(T_{1}, T_{2}\right) \in K\left(T_{1}, T_{2}\right)$ such that

$$
f\left(g\left(a_{1}^{\omega / k_{1}}, a_{2}^{\mu / k_{2}}\right), a_{1}^{\omega}, a_{2}^{\mu}\right)=0 .
$$

Since $a_{1}^{\omega / k_{1}} \in{ }^{*}{ }^{\circ} K-{ }^{*} K$ and $a_{2}^{\mu / k_{2}} \in{ }^{*} K-K$ are algebraically independent over $K$,

$$
f\left(g\left(T_{1}, T_{2}\right), T_{1}^{k_{1}}, T_{2}^{k_{2}}\right)=0
$$




\section{$\S 3$.}

Proof of Theorem for $m>2$ is essentially the same as that in Section 2. By induction on $i \in N$, we define iterated enlargements $K_{i}=\left(^{* i \cdots *_{2}{ }^{*}} K\right.$, $\left.{ }^{{ }_{i} \ldots \ldots{ }^{*} *_{2}} K, \cdots,{ }^{{ }_{i}} K\right)$ as follows. Let $K_{1}=\left({ }^{{ }_{1}} K\right) . \quad K_{i+1}$ is an enlargement of $\left(K_{i}, K\right)=\left({ }^{{ }^{*} i \cdots *^{* *_{1}}} K,{ }^{{ }_{i} \ldots{ }^{*} *^{*}+} K, \cdots,{ }^{{ }_{i}} K, K\right)$, i.e. $K_{i+1}={ }^{*_{i+1}}\left(K_{i}, K\right)=\left({ }^{*_{i+1}} K_{i},{ }^{{ }^{*} i+1} K\right)$. Let $\omega_{j} \in{ }^{{ }^{*} m \cdots *_{j+1{ }^{*} j}} N-{ }^{{ }^{*} m \cdots *_{j+1}} \boldsymbol{N}$ be divisible by all natural numbers. Let $\delta \in{ }^{*{ }^{*} \cdots{ }^{*} 1} K$ satisfy

$$
f\left(\delta, a_{1}^{\omega_{1}}, a_{2}^{\omega_{2}}, \cdots, a_{m}^{\omega_{m}}\right)=0 .
$$

Then by the same way as in Section 2 , there exist natural numbers $k_{1}, k_{2}, \cdots, k_{m}$ not more than $k$ such that $\delta \in K\left(a_{1}^{\omega_{1} / k_{1}}, \cdots, a_{m}^{\omega_{m} / k_{m}}\right)$. Since $a_{1}^{\omega_{1} / k_{1}}, \cdots, a_{m}^{\omega_{m} / k_{m}}$ are algebraically independent over $K$, there is a rational function $g\left(T_{1}, \cdots, T_{m}\right) \in K\left(T_{1}, \cdots, T_{m}\right)$ such that

$$
f\left(g\left(T_{1}, \cdots, T_{m}\right), T_{1}^{k_{1}}, \cdots, T_{m}^{k_{m}}\right)=0 .
$$

\section{REFERENCES}

[1] H. Davenport, D. J. Lewis and A. Schinzel, Polynomials of certain special types, Acta Arith., 9 (1964), 107-116.

[2] T. Kojima, Note on number theoretic properties of algebraic functions, Tohoku Math. J., 8 (1915), 24-37.

[3] A. Robinson and P. Roquette, On the finiteness theorem of Siegel and Mahler concerning diophantine equations, J. Number Theory, 7 (1975), 121-176.

[4] P. Roquette, Nonstandard aspects of Hilbert's irreducible theorem, Lecture Notes in Math., 498, 231-274.

[5] A. Schinzel, On Hilbert's irreducible theorem, Ann. Polon. Math., 16 (1965), 333340.

[6] - Selected topics on polynomials, Michigan 1982.

[ 7 ] S. Tung, On weak number theories, Thesis Illinois 1983.

[8] T. Skolem Einige Satze uber Polynome, Avhandlinger Norske Vid. Akad. Oslo, I Mat-Naturv. Kl. No. 4.

Department of Mathematics

Faculty of Science

Nagoya University

Chikusa-ku, Nagoya 464

Japan 